\title{
Least Action Trajectory in Neural Networks
}

\author{
Ellison C. Castro*, Bhazel Anne R. Pelicano \\ Department of Physical Sciences, University of the Philippines, Baguio City, Philippines \\ Email: *ellisonccastro@gmail.com
}

Received July 2013

\begin{abstract}
The study of complex networks had developed over the years to include systems such as traffic, predator-prey interactions, financial market, and even the world wide web. Complex network studies encompass biology, chemistry, physics, and even engineering and economics [1-6]. However, the dynamics of such complex networks are yet to be understood fully $[7,8]$. In this paper, we will be focusing mostly on the possible learning ability in a complex network. To do this, an optimization process is used via Wiener process [9,10]. It is apparent from the sample lattice shown that the final position was not a basis of the transition probability, or it was never used to calculate the probability, since the transition probability only considers the current position. The final point is reached because of the orientation of the edges, where each edge is facing the final point, an aspect of the nervous system (afferent and efferent nerves) [11-13]. No matter how random the orientation of the neurons is, each directs to the central nervous system for processing and is transmitted away for reaction.
\end{abstract}

Keywords: Neural Networks; Optimization; Wiener process

\section{Introduction}

Complex networks had been heavily used to model systems such as traffic [1-4], bankruptcy [14], social networking $[15,16]$, and protein-protein interaction $[17,18]$. While some of these systems are random in nature [19], others, such as human interactions (e.g. social networks) have learning capabilities. This behavior is similar to the brain [20], wherein, signals traveling from one neuron to another may opt to travel at the optimal path.

Finding the optimized path often leads us to the Lagrange equations of motion for classical dynamics [21]. However, quantum mechanics often resolve to take on optimization using path integral techniques [10], which, in principle, is similar to Lagrangian dynamics.

Hamilton's Principle and Lagrange equations of motion have been widely used to study the extremized, usually minimized, behavior and properties of mechanical systems (e.g. brachistochrone problem) [21]. However, the extremization of physical systems has been limited to classical systems. Several studies and researches have been reported of using the Lagrange and Hamiltonian dynamics to chaotic and stochastic systems $[22,23]$. In this paper, the extremized or least action path of Brownian is numerically simulated, to model propagation of neuronal signals.

\section{Methodology}

Before the numerical computation of the least-action

${ }^{*}$ Corresponding author. trajectory of the Brownian motion, the lattices (or the directed graphs) where the Brownian particle moves, are defined. To graphically show a regular lattice, each horizontal and vertical line is plotted within a for loop function, where the size and spacing of the lattice are concerned. As for the irregular lattice, each line shown is manually inputted. These methods show visual guides of the trajectory of the Brownian motion and do not affect the result.

Regardless of the type of lattice, each of them follows a form which defines each node and direction of the edge into the numerical computation. The nodes can be represented by $D G\{a, b\}=[x, y]$, where $D G$ is a matrix which contains two-element row matrices, having the elements $x$ and $y$, as its elements. The position of the row matrices is defined by $a$ and $b$ as the row and column position, respectively. The interpretations of the variables of $D G$ are as follows: the variable $a$ is the current node position of the Brownian particle, while $b$ is the node the Brownian particle can reach if it is in node $a$. The variables $x$ and $y$ are the Cartesian coordinates of node $b$. This definition of the coordinates of node $b$ will be useful numeric-wise and graphic-wise. In summary, if $D G\{a, b\}$ is not nil, then the Brownian particle at node $a$ can go to node $b$.

In the numerical process, the particle starts at node 1 for convenience. The program will search through all $D G\{1, b\}$ and pick all the elements having a value. Since the values are coordinates, they can be used to calculate 
the transition probability of the particle.

$$
W=\frac{1}{4 D\left(t-t_{0}\right)} \exp \left[-\frac{\left(x_{t}-x_{0}\right)^{2}}{4 D\left(t-t_{0}\right)}\right]
$$

The equation shows the transition probability of the Brownian particle in two dimensions, where $\boldsymbol{x}=\left(x_{1}, x_{2}\right)$ and $x^{2}=x_{1}^{2}+x_{2}^{2}$, and $D$ is the diffusion coefficient of the fluid. The time it takes for the particle to reach a specific position (i.e. $t-t_{0}$ ) is assumed to be unity. Each $D G$ that has a value will have a corresponding transition probability. These probabilities are then stored in a stochastic matrix for normalization. The columns of the stochastic matrix correspond to the Brownian particle's initial node position, whereas the rows correspond to the possible future positions of the particle. From here, the comparison of the probabilities will start in a specific column and then through the rows of the column. This will return the element position having the highest probability, which in turn can be translated into the next current node. The process repeats until the Brownian particle reaches the final node and then the whole process is redone for Monte Carlo simulation.

\section{Results}

To model a more realistic neural network, an irregular design is used (e.g. Figure 1). This is a directed graph of a neural network where each node represents the cell body and each directed edge defines the orientation of axons (the signal can be transmitted in the direction of the arrow).

In the optimized path of the signal (Figure 2), the path do not pass through points other than the almost horizontal line connecting the initial and final position.

It is apparent from both lattices (Figures 2 and 3) that

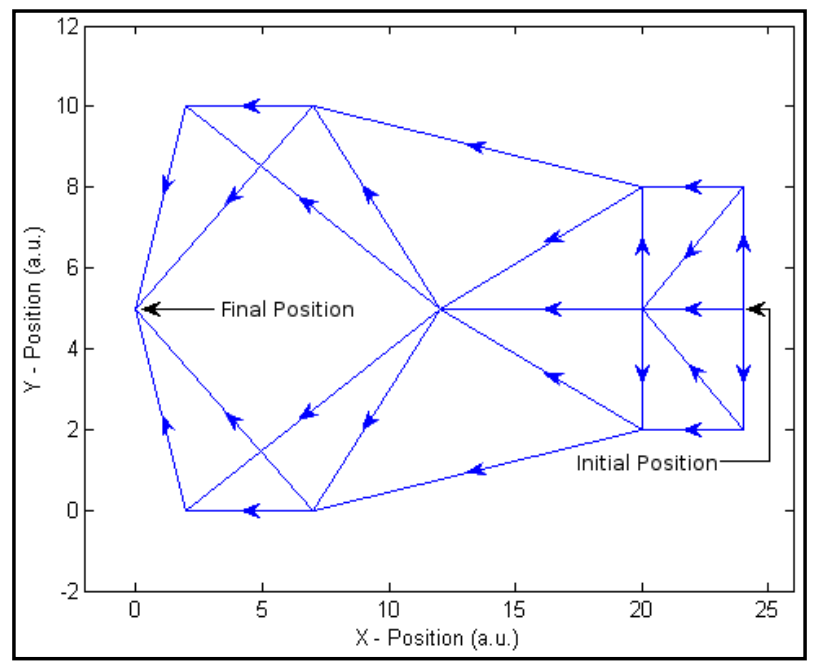

Figure 1. Example of a directed graph for an irregular lattice.

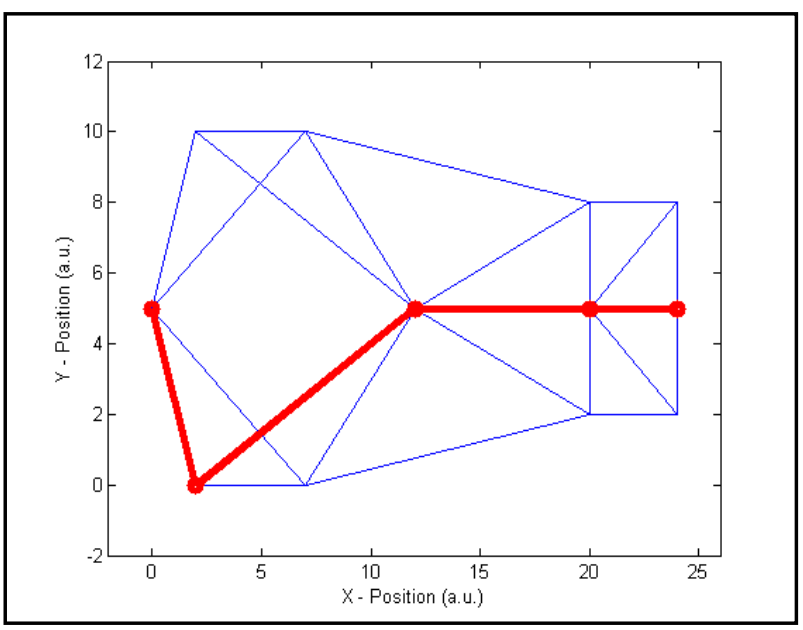

Figure 2. Optimal path for an irregular lattice.

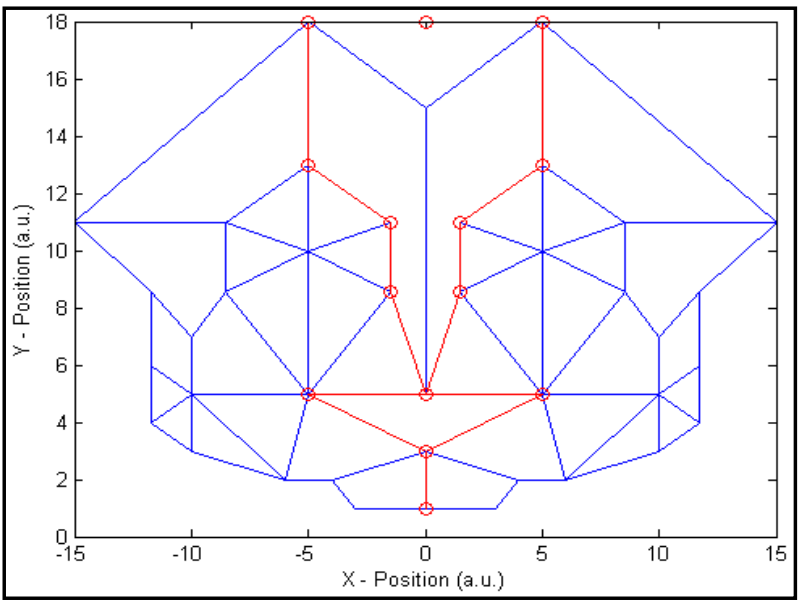

Figure 3. Simplified optimal path from the cortex to the eyes.

the final position was not a basis of the transition probability, nor was it used to calculate the probability. This is because the transition probability only considers the current position, which means there is no correlation between "steps" as the signal moves from node to node. The final point is reached because of the orientation of the edges, where each edge is facing the final point, an aspect of the nervous system (afferent and efferent nerves [11-13]). No matter how random the orientation of the neurons, each directs to the central nervous system for processing and is transmitted away for reaction.

The study by De Marco Garcia, et al., on the identification of the muscle nerve trajectory has shown that signals, differentiated by dyes, do not diffuse onto all networks [24]. This model may be able to simulate what is going on in the said-system.

\section{Summary and Conclusions}

Using the dependence of the Brownian motion's action 
to the transition probability, the trajectory of least action for the motion can be described. The optimized path in free space does not depend on the frequency of the particle to pass through a certain point, but depends on the most probable point in space in relation to the particle's current position. Even though the transition probability is independent of the history of the Brownian motion, the path of least action is indirectly affected by the initial positions.

Brownian motion in a neural network still has a classical characteristic, i.e. the trajectory most likely follows a straight line from the starting position to the designated point. Whereas, the installation of the condition of a final point never appears in the transition probability calculation. It appears to be that it is inherent to the transition probability itself that the Brownian motion is subject to follow a horizontal of vertical displacement. The final position can then be reached if the Brownian motion approaches the point, which can be done by orienting the edges of the directed graph.

\section{REFERENCES}

[1] L. Zhao, Y.-C. Lai, K. Park and N. Ye, "Onset of Traffic Congestion in Complex Networks," Physical Review E, Vol. 71, No. 2, 2005. http://dx.doi.org/10.1103/PhysRevE.71.026125

[2] B. Tadic, S. Thurner and G. J. Rodgers, "Traffic on Complex Networks: Towards Understanding Global Statistical Properties from Microscopic Density Fluctuations," Physical Review E, Vol. 69, No. 3, 2004.

[3] G. Yan, T. Zhou, B. Hu, Z.-Q. Fu and B.-H. Wang, "Efficient Routing on Complex Networks," Physical Review E, Vol. 73, No. 4, 2006. http://dx.doi.org/10.1103/PhysRevE.73.046108

[4] C. Daganzo, "The Cell Transmission Model, Part 2: Network Traffic,” Transportation Research Part B: Methodological, Vol. 29, No. 2, 1995, pp. 79-93. http://dx.doi.org/10.1016/0191-2615(94)00022-R

[5] S. Allesina and M. Pascual, "Network Structure, Predator-Prey Modules, and Stability in Large Food Webs," Theoretical Ecology, Vol. 1, No. 1, 2008. http://dx.doi.org/10.1007/s12080-007-0007-8

[6] R. Milo, S. Shen-Orr, S. Itzkovitz, N. Kashtan, D. Chklovskii and U. Alon, "Network Motifs: Simple Building Blocks of Complex Networks,” Science, Vol. 298, No. 5594, 2002, pp. 824-827. http://dx.doi.org/10.1126/science.298.5594.824

[7] S. Strogatz, "Exploring Complex Networks,” Nature, Vol. 410, 2001, pp. 268-276. http://dx.doi.org/10.1038/35065725

[8] S. Boccaletti, V. Latora, Y. Moreno, M. Chavez and D.-U. Hwang, "Complex Networks: Structure and Dynamics," Vol. 424, No. 4-5, 2006, pp. 175-308. http://dx.doi.org/10.1016/j.physrep.2005.10.009

[9] L. S. Schulman, "Brownian Motion and the Wiener Inte- gral; Kac's Proof,” Techniques and Applications of Path Integration, John Wiley \& Sons Inc., Hoboken, 1981, pp. 53-64.

[10] M. Chaichian and A. Demichev, "Wiener's Treatment of Brownian Motion: Wiener Path Integrals,” Path Integrals in Physics: Stochastic Processes and Quantum Mechanics, Vol. 1, IOP Publishing, Bristol, 2001, pp. 22-38.

[11] R. Bernstein and S. Bernstein, "Biology,” Wm. C. Publishers, Dubuque, 1996.

[12] P. Raven and G. Johnson, "Biology," 6th Edition, McGraw-Hill Companies, New York, 2002.

[13] N. Campbell, J. Reece and L. Mitchell, “Biology,” 5th Edition, Benjamin Cummings, San Francisco, 1999.

[14] M. D. Odom and R. Sharda, "A Neural Network Model for Bankruptcy Prediction,” Neural Networks, 1990 IJCNN International Joint Conference on, Vol. 2, 1990, pp. 163168. http://dx.doi.org/10.1109/IJCNN.1990.137710

[15] A. Grabowski, "Interpersonal Interactiona and Human Dynamics in a Large Social Network,” Physical A, Vol. 385, pp. 363-369.

[16] D. J. Watts and S. H. Strogatz, "Collective Dynamics of 'Small-World' Networks,” Nature, Vol. 393, 1998, pp. 440-442. http://dx.doi.org/10.1038/30918

[17] Z. C. Lou, Y. H. Lai, L. L. Chen, X. Zhou, Z. Dai and X. Y. Zou, "Identification of Human Protein Complexes from Local Sub-Graphs of Protein-Protein Interaction Network Based on Random Forest with Topological Structure Features," Analytica Chimica Acta, Vol. 718, 2012, pp. 32-41. http://dx.doi.org/10.1016/j.aca.2011.12.069

[18] A. Sharma, S. Costantini and G. Colonna, "The ProteinProtein Interaction Network of the Human Sirtuin Family," Biochemica et Biophysica Acta. Article in Press.

[19] A. L. Barabasi and R. Albert, "Emergence of Scaling in Random Networks,” Science, Vol. 286, No. 5439, 1999, pp. 509-512. http://dx.doi.org/10.1126/science.286.5439.509

[20] G. J. Ortega, R. G. Sola and J. Pastor, “Complex Network Analysis of Human ECoG Data,” Neuroscience Letters, Vol. 447, 2008, pp. 129-133. http://dx.doi.org/10.1016/j.neulet.2008.09.080

[21] S. Thornton and J. Marion, "Classical Dynamics of Particles and Systems,” Brooks/Cole, Thomson Learning, Belmont, 2004.

[22] N. Mordant, J. Delour, E. Leveque, A. Arneodo and J. F. Pinton, "Long Time Correlations in Lagrangian Dynamics: A Key toIntermittency in Turbulence,” Phys. Rev. Lett., Vol. 89, No. 254502, 2002. http://dx.doi.org/10.1103/PhysRevLett.89.254502

[23] N. Mordant, E. Leveque and J. F. Pinton, "Experimental and Numerical Study of the Lagrangian Dynamics of High Reynolds Turbulence," New Journal of Physics, Vol. 6, No. 116, 2004.

[24] N. De Marco Garcia and T. Jessell, "Early Motor Neuron Pool Identity and Muscle Nerve Trajectory Defined by Postmitotic Restrictions in Nkx6.1 Activity,” Neuron, Vol. 57, No. 2, 2008. 